\title{
miR-454-3p promotes of human glioma cell growth by targeting EGR3
}

\author{
GUOLIANG SHEN, JIAN WU, ZHAOHUI MOU, DANLONG CHEN, LI LIU and XING LI
}

Department of Neurosurgery, The First People's Hospital of Taizhou, Taizhou, Zhejiang 318020, P.R. China

Received December 13,2018; Accepted August 16, 2019

DOI: $10.3892 /$ etm.2019.8048

\begin{abstract}
Gliomas are the most common primary brain tumors in adults and are associated with high mortality rates. In the present study, the aim was to evaluate the role of miR-454-3p in the pathogenesis of human glioma and to explore the underlying mechanism. Reverse transcription-quantitative PCR was performed to compare the expression levels of miR-454-3p in glioma and adjacent normal tissue. The effects of miR-454-3p on cell proliferation was tested by combining MTT and colony formation assays. Dual-luciferase assay was used to identify the target gene of miR-454-3p. The results showed that miR-454-3p was upregulated in glioma tissues where it exerts a positively regulatory role on cell growth. Dual-luciferase assay confirmed Early Growth Response 3 (EGR3) to be a target for miR-454-3p. Overexpression of EGR3 in glioma cells was found to impair miR-454-3p mimic-induced cell proliferation. These results suggested that upregulated miR-454-3p served an important role in glioma tumorigenesis by targeting EGR3, which provided valuable insights into the underlying mechanism of the disease that may lead to possible novel therapeutic strategies.
\end{abstract}

\section{Introduction}

Glioma is a primary brain tumor in the central nervous system that is highly aggressive. Although the morbidity of glioma is relatively low (accounting for $2 \%$ in all adult malignancies), the number of newly diagnosed patients is increasing (1). Since gliomas have tendencies to invade surrounding tissues, current treatment strategies are ineffective and consequently the prognosis for patients remains poor (2). Therefore, there is currently an urgent need to explore the pathological mechanism of this disease to devise new therapeutic interventions.

With the advancing knowledge and understanding of microRNAs (miRNAs), miRNAs are now known as an important class of post-transcriptional regulators of gene expression

Correspondence to: Dr Jian Wu, Department of Neurosurgery, The First People's Hospital of Taizhou, 218 West Hengjie Road, Taizhou, Zhejiang 318020, P.R. China

E-mail: wujianaiJW@163.com

Key words: microRNA-454-3p, glioma, cell proliferation, early growth response 3 by translational repression and mRNA breakdown (3-5). It has been shown to participate in a variety of biological processes, including cell proliferation, differentiation, migration, apoptosis, cell-cycle regulation and invasion, development and metabolism by targeting the 3'-untranslated regions (3'UTR) of target genes (6). Accumulating evidence have also indicated aberrant expression of miRNAs were found in a number of diseases, particularly in cancers (7). Indeed, a wide range of miRNAs have been reported to function as biomarkers for diagnosis and prognosis of human glioma (8). Decreased miR-374a expression was associated with glioma progression (9), whereas increased expressions of miRNA-21, miR-128 or miR-342-3p were positively correlated with histopathological grades of glioma $(10,11)$. In fact, >97 miRNAs were found to be aberrantly expressed through miRNA microarrays and involved in the pathogenesis of glioma through major signaling pathways (12). However, the role of miR-454-3p and underlying mechanism in glioma remains poorly understood.

Early growth response 3 (EGR3) is a member of the EGR family of $\mathrm{C} 2 \mathrm{H} 2$-type zinc-finger proteins (13). It regulates gene transcription and has been found to be involved in multiple biological processes, including the development of muscle, lymphocyte and neurons, in addition to the growth, migration, invasion and metastasis of cancer cells (14-16). Therefore, associations between abnormal EGR3 expression and schizoaffective disorder, schizophrenia, systemic autoimmunity, and a variety of cancers has been previously demonstrated (14-16). In hepatocellular carcinoma, downregulation of EGR3 expression of promoted cell proliferation by upregulating Fas ligand expression (17). In contrast, reduction in EGR3 expression by KH-type splicing regulatory protein suppressed cell invasion and metastasis in non-small cell lung cancer (NSCLC) (14). These previous findings suggest that the function of EGR3 may differ depending on the type of cancer in question.

In the present study, the aim is to elucidate the potential role of miR-454-3p in glioma and explore the potential mechanism. The results can deepen the understanding on the pathogenesis of glioma which may provide novel molecular targets for treating this disease.

\section{Materials and methods}

Ethics statement. The present study was approved by the Research Ethics Committee of The First People's Hospital of 
Taizhou. All patients provided written informed consent prior to enrollment in the study.

Tissue specimens. Clinical samples were obtained from 40 patients (sex, 20 males and 20 females; age range, 28-71 years; mean age, $58.5 \pm 14.1$ years) who were diagnosed with glioma according to World Health Organization (WHO) classification without preoperative radiation or chemotherapy, by surgery at the The First People's Hospital of Taizhou from January 2015 to December 2016 (Taizhou, China). Patients who had received neoadjuvant or adjuvant therapy were excluded from the present study. Tumors and adjacent non-tumor intracranial tissue samples were collected and immediately frozen in liquid nitrogen.

Cell line and culture. Human glioma cell lines U138MG (cat.no. ATCC ${ }^{\circledR} \mathrm{HTB}-16^{\mathrm{TM}}$ ) and 251 (cat.no. ATCC ${ }^{\circledR} \mathrm{HTB}-17$ ), together with $293 \mathrm{~T}$ cells (cat. no. ATCC ${ }^{\circledR}$ CRL-11268 ${ }^{\mathrm{TM}}$ ) were purchased from the American Type Culture Collection. And normal human astrocytes (NHA; cat. no. BNCC341796), A172 (cat. no. BNCC341782) cells were purchased from BeNa culture collection. U138MG, NHA, A172 and 293T cells were cultured in DMEM (Gibco; Thermo Fisher Scientific, Inc.) supplemented with $10 \%$ fetal bovine serum (Sigma-Aldrich; Merck KGaA) and penicillin (100 IU/ml)/streptomycin $(100 \mu \mathrm{g} / \mathrm{ml})$, whilst U251 cells were cultured in RPMI-1640 media (Gibco; Thermo Fisher Scientific, Inc.), supplemented with $10 \%$ fetal bovine serum (Sigma-Aldrich; Merck KGaA) and penicillin $(100 \mathrm{IU} / \mathrm{ml}) /$ streptomycin $(100 \mu \mathrm{g} / \mathrm{ml})$ in a humidified incubator containing $5 \% \mathrm{CO}_{2}$ at $37^{\circ} \mathrm{C}$.

Cell transfection with mimics, inhibitor, siRNA and plasmids. miR-454-3p mimic and its negative control (NC), inhibitor of miR-454-3p and its negative control (NC-in), siRNA for EGR3 (siEGR3) and its negative control (siNC) were synthesized by Shanghai GenePharma Co., Ltd., with their respective sequences listed in Table II. For the overexpression of EGR3, the coding sequence for EGR3 was amplified from NHA cells using KOD-Plus-Neo Polymerase (cat. no. KOD-401; Toyobo Life Science) using the forward primer containing a $\mathrm{BamHI}$ restriction site, 5'-GGGGATCCATGACCGGCAAACTCG CCGAGAAGC-3' and the reverse primer, containing a EcoRI restriction site, 5'-GGGAATTCTCAGGCGCAGGTGGTG ACCACGG-3' and then subcloned into the pcDNA3.1 plasmid (Invitrogen; Thermo Fisher Scientific, Inc.). The mimics (50 $\mathrm{nM})$ and inhibitor (100 $\mathrm{nM})$ of miR-454-3p, siEGR3 $(100 \mathrm{nM})$, plasmids $(1 \mathrm{ng} / \mu \mathrm{l})$ and their negative controls were transfected into cells using Lipofectamine ${ }^{\circledR} 3000$ (Invitrogen; Thermo Fisher Scientific, Inc.) according to manufacturer's protocol.

Reverse transcription-quantitative PCR (RT-qPCR). Total RNA was extracted from the cells or tissues using mirVana ${ }^{\mathrm{TM}}$ miRNA Isolation Kit (Ambion; Thermo Fisher Scientific, Inc.) according to manufacturer's protocol. To measure the levels of miR-454-3p expression, TaqMan ${ }^{\mathrm{TM}}$ MicroRNA Reverse Transcription Kit (Applied Biosystems; Thermo Fisher Scientific, Inc.) were used to perform reverse transcription according to manufacturer's protocol. To determine the mRNA levels of EGR3, total RNA was reverse transcribed into cDNA using PrimeScript ${ }^{\mathrm{TM}}$ RT reagent Kit with gDNA Eraser (Takara Biotechnology Co., Ltd.). qPCR was performed using SYBR ${ }^{\circledR}$ PrimeScript $^{\mathrm{TM}}$ RT-PCR kit (Takara Biotechnology Co., Ltd.) according to manufacturers' protocols using Applied Biosystems ${ }^{\circledR} 7500$ Real-Time PCR Systems (Applied Biosystems; Thermo Fisher Scientific, Inc.) under the following thermocycling conditions: Initial denaturation at $95^{\circ} \mathrm{C}$ for $10 \mathrm{~min}$, followed by 40 cycles of $95^{\circ} \mathrm{C}$ for $5 \mathrm{sec}$ and $60^{\circ} \mathrm{C}$ for $30 \mathrm{sec}$. U6 snRNA and GAPDH were used as reference genes for miR-454-3p and EGR3 respectively. Relative expression levels were calculated using the $2^{-\Delta \Delta \mathrm{Cq}}$ method (18). All PCRs were performed in triplicate for analysis and the sequences of primers used were listed in Table III.

Cell viability assay. Cell growth was measured using MTT (Sigma-Aldrich; Merck KGaA) and colony formation assays. For MTT assays, approximately 3,000 cells/well were seeded into 96-well plates, following which $20 \mu \mathrm{l}$ MTT dissolved in PBS $(5 \mathrm{mg} / \mathrm{ml})$ was added to each well at indicated times and incubated for a further $4 \mathrm{~h}$. The supernatant was then carefully removed and $150 \mu \mathrm{l}$ DMSO was added to each well and plates were subsequently shaken for $10 \mathrm{~min}$ to dissolve the formazan crystals. Optical density at $490 \mathrm{~nm}$ was then measured per well using a Thermo Scientific Multiskan (Thermo Fisher Scientific, Inc.).

For colony formation assays, 1,000 cells were plated into each well of a 6-well plate and cultured for 14 days. Colonies were subsequently fixed using $10 \%$ formaldehyde for $5 \mathrm{~min}$ at room temperature (RT) and stained using $0.1 \%$ crystal violet for $30 \mathrm{sec}$ at RT. The numbers of colonies were counted for each condition using light microscopy. Each experiment was repeated three times independently.

Prediction targets of miR-454-3p. The targets of miR-454-3p were predicted using the TargetScan website (Version 7.1; http://www.targetscan.org/vert_71/) (19).

Dual-luciferase reporter assay. For dual-luciferase reporter assays, 4,000 293T cells were seeded into 96-well plates and pMirGLO plasmids ( $1 \mathrm{ng} / \mu \mathrm{l})$ (Promega Corporation) encoding wild-type or mutant 3'UTR of EGR3 were co-transfected into cells with $20 \mathrm{nM}$ either miR-454-3p mimics or NC using Lipofectamine 3000. Firefly and Renilla luciferase activities were assayed $24 \mathrm{~h}$ after transfection using Dual-Luciferase ${ }^{\circledR}$ Reporter Assay System (Promega Corporation) according to manufacturer's protocols. Firefly luciferase activities were normalized to Renilla luciferase activities. Each experiment was repeated three times independently.

Western blot analysis. For western blotting, tissues were homogenized in RIPA buffer (Cell Signaling Technology, Inc.) from which protein samples were isolated. After quantification using Pierce ${ }^{\circledR}$ Bicinchoninic Acid Protein Assay Kit (Thermo Fisher Scientific, Inc.), $40 \mu \mathrm{g}$ total protein samples per lane were subjected to $10 \%$ SDS-PAGE and were subsequently transferred to a PVDF membrane. The membranes were then blocked with $5 \%$ skimmed milk dissolved in PBS for $1 \mathrm{~h}$ at room temperature followed by incubation with primary antibodies against EGR3 (1:100; cat. no. sc-390967; Santa Cruz Biotechnology, Inc.) and GAPDH (1:500; cat. no. D16H11; Cell 
Signaling Technology, Inc.) at $4^{\circ} \mathrm{C}$ overnight. The membranes were subsequently washed using tris-buffered saline with Tween-20 $(0.05 \%)$ three times for $5 \mathrm{~min}$ each and subsequently incubated in horseradish peroxidase-conjugated secondary antibody (1:2,000; cat. no. 7076 for EGR3 detection and cat. no. 7074 for GAPDH detection; Cell Signaling Technology, Inc.) for $1 \mathrm{~h}$ at room temperature. Protein bands were visualized using an enhanced ECL-kit (Thermo Fisher Scientific, Inc.) and captured using the ChemiDoc ${ }^{\mathrm{TM}} \mathrm{XRS}+$ System (Version: 5.2.1; Bio-Rad Laboratories, Inc.). Bands were quantified using ImageJ (National Institutes of Health) and normalized to GAPDH.

Statistical analysis. Statistical analyses were performed using the GraphPad Prism 6 software (GraphPad Software, Inc.) and data were presented as mean \pm SD. Differences between two groups were analyzed using Student's t-test, whereas Tukey's multiple comparisons test after ANOVA was applied for $>3$ groups. And the survival curve was constructed using the Kaplan-Meier method and compared using the log-rank test, high- and low-expressing groups were divided according to the median expression of miR-454-3p. $\mathrm{P}<0.05$ was considered indicate a statistically significant difference.

\section{Results}

miR-454-3p is upregulated in human glioma tissues. In order to investigate the role of miR-454-3p in human glioma pathogenesis, expression levels of miR-454-3p were compared between glioma and adjacent non-tumor samples in intracranial tissues from 40 patients using RT-qPCR. The baseline patient and tumor characteristics were summarized in Table I. The expression levels of miR-454-3p were associated with the tumor size $(\mathrm{P}=0.024)$ and WHO stage $(\mathrm{P}=0.011)$. As shown in Fig. 1A, miR-454-3p expression in glioma tissues $(0.54 \pm 0.03, \mathrm{n}=40)$ was significantly higher compared with that in adjacent normal tissues $(1.10 \pm 0.04, n=40)$. Overall survival analysis revealed that patients with higher expression levels of miR-454-3p expression were associated with significantly shorter overall survival (median survival: 687 vs. 1,143 days, $n=40$, Fig. 1B). The results indicated that miR-453-3p may be involved in glioma tumorigenesis. To determine the function of miR-454-3p further in glioma cells, the expression levels of miR-454-3p were measured in U251, U138MG and A172 glioma cell lines and NHA cells. When compared with NHA cells, miR-454-3p expression was found to be significantly increased in glioma cells, with the magnitude the greatest in U251 cells (Fig. 1C).

miR-454-3p promotes human glioma cell proliferation. MTT and colony formation assay were performed to determine the effects of miR-454-3p on cell proliferation. Considering the different expression levels of miR-454-3p in U138MG and U251 cells, U138MG and U251 cells were first transfected with miR-454-3p mimics or inhibitor, respectively. The expression levels of miR-454-3p were significantly increased in U138MG cells following transfection with miR-454-3p mimics compared with its corresponding negative control (NC); in contrast, miR-454-3p expression was significantly reduced in $\mathrm{U} 251$ cells following transfection with the
Table I. Association between clinicopathological features and expression of miR-454-3p.

\begin{tabular}{|c|c|c|c|c|}
\hline \multirow[b]{2}{*}{ Characteristic } & \multirow[b]{2}{*}{$\mathrm{n}$} & \multicolumn{2}{|c|}{$\begin{array}{c}\text { Relative } \\
\text { miR-454-3p } \\
\text { expression }\end{array}$} & \multirow[b]{2}{*}{ P-value } \\
\hline & & Low & High & \\
\hline Age & & & & 0.301 \\
\hline$<45$ & 16 & 6 & 10 & \\
\hline$\geq 45$ & 24 & 13 & 11 & \\
\hline Sex & & & & 0.113 \\
\hline Female & 20 & 12 & 8 & \\
\hline Male & 20 & 7 & 13 & \\
\hline Tumor size & & & & 0.024 \\
\hline$<5 \mathrm{~cm}$ & 22 & 14 & 8 & \\
\hline$\geq 5 \mathrm{~cm}$ & 18 & 5 & 13 & \\
\hline Peritumoral brain edema & & & & 0.342 \\
\hline$<1 \mathrm{~cm}$ & 20 & 8 & 12 & \\
\hline$\geq 1 \mathrm{~cm}$ & 20 & 11 & 9 & \\
\hline WHO stage & & & & 0.011 \\
\hline I & 9 & 8 & 1 & \\
\hline II & 8 & 5 & 3 & \\
\hline III & 8 & 2 & 6 & \\
\hline IV & 15 & 4 & 11 & \\
\hline
\end{tabular}

miR, microRNA; WHO, world health organization.

miR-454-3p inhibitor compared with its corresponding NC-in (n=3; Fig. 2A). MTT assay showed that miR-454-3p overexpression significantly increased U138MG cell viability, whereas miR-454-3p knockdown significantly reduced U251 cell viability ( $\mathrm{n}=5$; Fig. 2B). Colony formation assay showed consistent results (Fig. 2C) U138MG cells transfected with miR-454-3p mimics exhibited significantly more colonies compared with those transfected with NC (26 \pm 3 vs. $117 \pm 9$, $\mathrm{n}=3$ ), whilst U251 cells transfected with miR-454-3p inhibitor resulted in significantly decreased colony numbers $(114 \pm 9$ vs. $28 \pm 8, n=3)$. These results suggested that the overexpression of miR-454-3p enhanced human glioma cell growth in vitro.

miR-454-3p directly targets the 3'-UTR of the EGR3 gene. Potential target genes of miR-454-3p were next predicted using the TargetScan Database. As shown in Fig. 3A, a potential binding site on the 3'UTR of EGR3 was detected for miR-454-3p. To verify this, a dual-luciferase reporter assay we performed using plasmids encoding the wild-type or mutant sequence of the EGR3 3'UTR. The relative luciferase activity of cells co-transfected with wild-type EGR3 3'UTR and miR-454-3p mimics was significantly lower compared with that co-transfected with NC and wild-type EGR3 3'UTR $(0.40 \pm 0.04$ vs. $1.00 \pm 0.03, n=3)$. However, the relative luciferase activity of cells co-transfected with miR-454-3p mimics and the mutant sequence of EGR3 3'UTR showed no marked difference compared with that co-transfected with $\mathrm{NC}$ and mutant sequence of the EGR3 3'UTR $(0.98 \pm 0.06$ vs. $1.0 \pm 0.06$; 
Table II. Sequences.

Targets

Sequences $\left(5^{\prime}-3^{\prime}\right)$

Duplex of mimics of miR-454-3p

UAGUGCAAUAUUGCUUAUAGGGU

Duplex of NC

CCUAUAAGCAAUAUUGCACUAUU

UUCUCCGAACGUGUCACGUTT

Inhibitor of miR-454-3p

ACGUGACACGUUCGGAGAATT

$\mathrm{NC}$-in

ACCCUAUAAGCAAUAUUGCACUA

siEGR3

UUGUACUACACAAAAGUACUG

siNC

AGAUCCACCUCAAGCAAAAUU

AAUUCUCCGAACGUGUCACGU

NC, negative control; miR, microRNA; EGR3, early growth response 3 .

Table III. Primer sequences.

\begin{tabular}{ll}
\hline Primers & \multicolumn{1}{c}{ Sequences $\left(5^{\prime}-3^{\prime}\right)$} \\
\hline miRNA reverse transcription primer & GTCGTATCCAGTGCAGGGTCCGAGGTATTCGCACTGGATACGACACCCTA \\
miRNA-454-3p-F & ACCCTATCAATATTGTCTCTGC \\
miRNA-454-3p-R & GCGAGCACAGAATTAATACGAC \\
U6-F & CTCGCTTCGGCAGCACA \\
U6-R & AACGCTTCACGAATTTGCGT \\
EGR3-F & TTCGCTTTCGACTCTCC \\
EGR3-R & CTCCGAGTAGAGATCGC \\
GAPDH-F & CCACCCATGGCAAATTCC \\
GAPDH-R & CAGGAGGCATTGCTGATGAT \\
\hline
\end{tabular}

miRNA, microRNA; EGR3, early growth response 3; F, forward; R, reverse.

n=3; Fig. 3A). According to RT-qPCR analysis, the expression levels of EGR3 mRNA in U138MG cells transfected with miR-454-3p mimics was significantly decreased compared with cells transfected with NC. On the contrary, transfection with the miR-454-3p inhibitor significantly reduced EGR3 expression in U251 cells compared with cells transfected with NC-in (Fig. 3B). Transfection with miR-454-3p mimics significantly reduced the expression level of EGR3 proteins compared with those transfected with $\mathrm{NC}$ in U138MG cells $(0.13 \pm 0.01$ vs. $0.92 \pm 0.04, n=3$ ), whilst transfection with the miR-454-3p inhibitor significantly increased EGR3 protein expression in U251 cells $(0.79 \pm 0.02$ vs. $0.20 \pm 0.01, n=3)$ compared with those transfected with NC-in (Fig. 3C). These results suggest EGR3 to be a target gene of miR-454-3p.

EGR3 is downregulated in glioma tissues and its overexpression inhibits glioma cell proliferation. To reveal the function and significance of EGR3 in glioma tumors, the expression levels of EGR3 in glioma and adjacent normal tissues were tested using RT-qPCR and western blot analysis. The expression of EGR3 mRNA $(0.67 \pm 0.03$ vs. $0.35 \pm 0.02, n=40)$ and protein $(0.75 \pm 0.04$ vs. $0.27 \pm 0.02, n=3)$ in glioma tissues were significantly decreased compared with adjacent normal tissues (Fig. 4A and B). The effects of EGR3 on glioma cell proliferation were investigated further using MTT and colony formation assays. EGR3 was first overexpressed in U138MG cells by transfection with plasmids expressing EGR3 and knocked down in U251 cells by siRNA transfection. Transfection efficiency was confirmed using RT-qPCR and western blot analyses (n=3, Fig. 4C and D). EGR3 overexpression significantly reduced the viability of U138MG cells ( $n=5$, Fig. 4E), in addition to significantly reducing the colony number of U138MG cells compared with cells transfected with negative control vector $(85 \pm 3$ vs. $36 \pm 3$; n=3; Fig. $4 \mathrm{~F})$. In contrast, following transfection with siEGR3, U251 cell viability was significantly increased $(n=5)$ and colony number was also significantly higher compared with those transfected

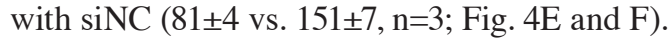

EGR3 is involved in miR-454-3p-induced elevation of glioma cell proliferation. Based on the findings already obtained from the present study, it was hypothesized that miR-454-3p may promote glioma cell proliferation of by targeting and regulating EGR3 expression. U138MG and U251 cells were either transfected with miR-454-3p mimics only or co-transfected with miR-454-3p mimics and EGR3 plasmids. Transfection with miR-454-3p mimics significantly reduced EGR3 expression, which was reversed by co-transfection with EGR3 plasmids (n=3, Fig. 5A and B). Besides, the results of MTT and colony formation assays showed that transfection with 
A

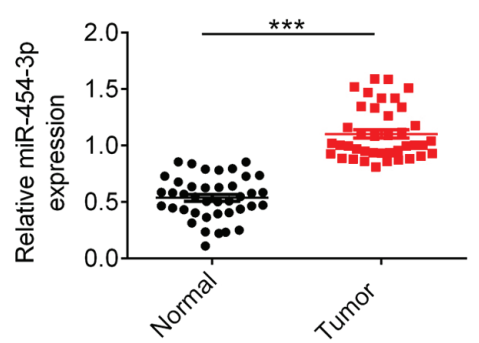

B

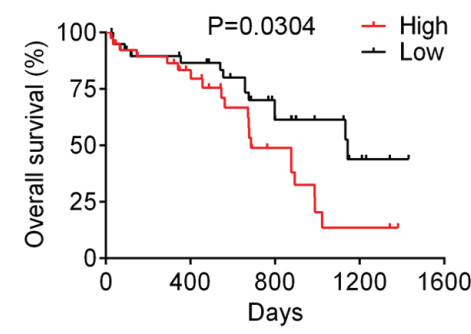

C

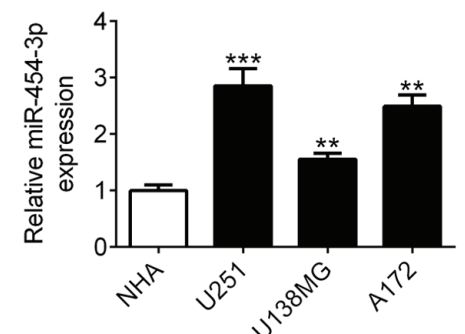

Figure 1. Expression levels of miR-454-3p was upregulated in glioma. (A) Relative expression levels of miR-454-3p in glioma and adjacent normal tissues were determined using RT-qPCR. ${ }^{* * * *} \mathrm{P}<0.001$. (B) Comparison of overall patient survival between the high miR-454-3p expression and low miR-454-3p expression groups. The high miR-454-3p expression group exhibited significantly shorter survival compared with that of the low miR-454-3p expression of group. $\mathrm{P}=0.0304$. (C) Relative miR-454-3p expression in cell lines were determined using RT-qPCR. ${ }^{* *} \mathrm{P}<0.01$ and ${ }^{* * * *} \mathrm{P}<0.001$ vs. NHA cells. RT-qPCR, reverse transcription-quantitative PCR; miR, microRNA; NHA, normal human astrocyte cells.
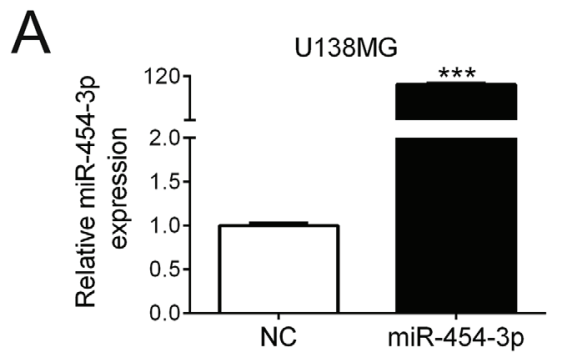

B
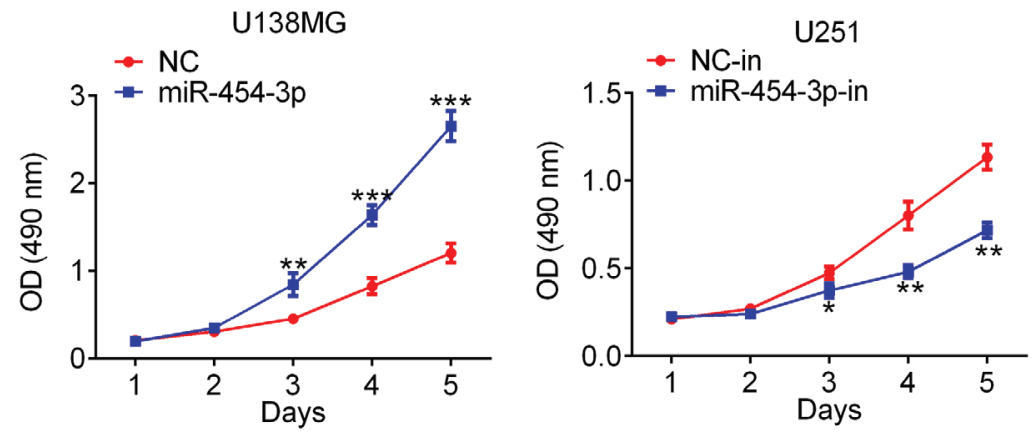

C
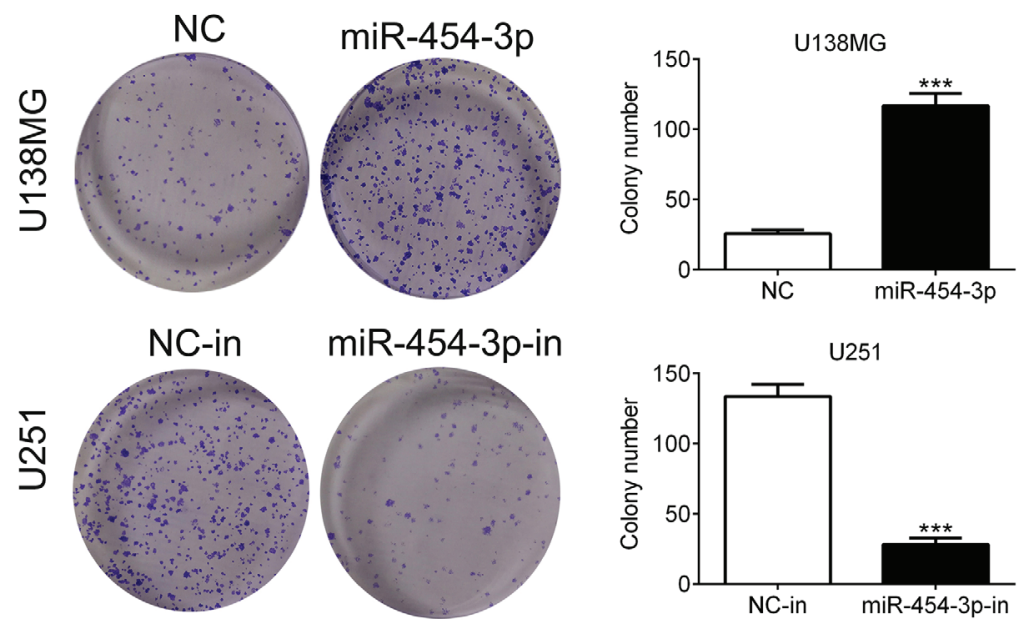

Figure 2. miR-454-3p promoted cell proliferation in glioma cell lines. (A) Expression levels of miR-454-3p were determined using reverse transcription-quantitative PCR in U138MG cells transfected with miR-454-3p mimics and in U251 cells transfected with miR-454-3p inhibitor. (B) MTT assay were performed to assess viability in U138MG cells transfected with miR-454-3p mimics and U251 cells transfected with miR-454-3p inhibitor. (C) Colony formation assay was performed to measure the growth of U138MG cells transfected with miR-454-3p mimics and U251 cells transfected with miR-454-3p inhibitor. Representative images of crystal violet-stained cell colonies are shown. ${ }^{*} \mathrm{P}<0.01,{ }^{* *} \mathrm{P}<0.05$ and ${ }^{* * * *} \mathrm{P}<0.001$ vs. NC or NC-in. NC, negative control; miR, microRNA; OD, optical density. 
A

hsa-miR-454-3p 3' UGGGAUAUUCGUUAUAACGUGAU 5'
EGR3 3' UTR-wt 5'...GUAAUGUUGUACCAUUUGCACUG...3'

EGR3 3' UTR-mut 5'...GUAAUGUUGUACCAUAACGUGAG...3'
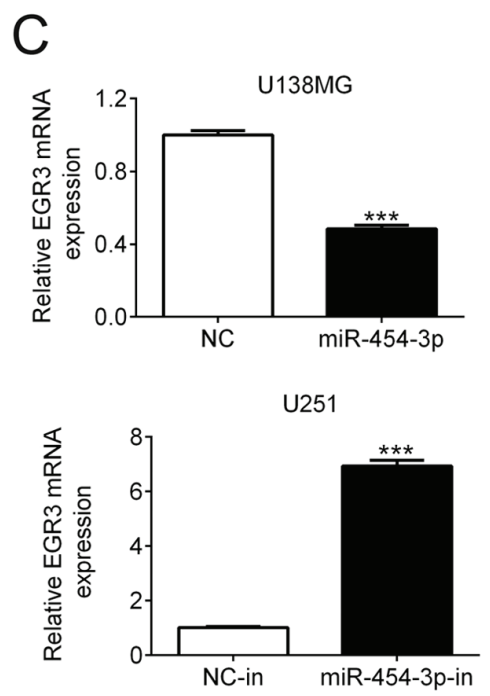

$\mathrm{D}$

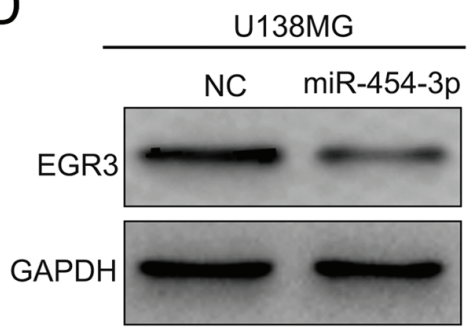

U251

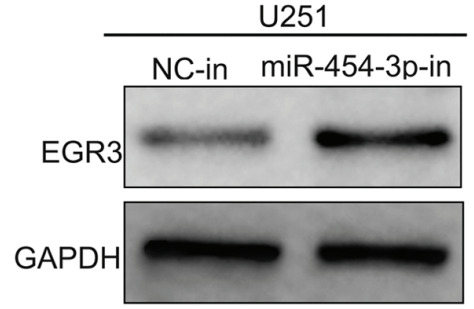

B

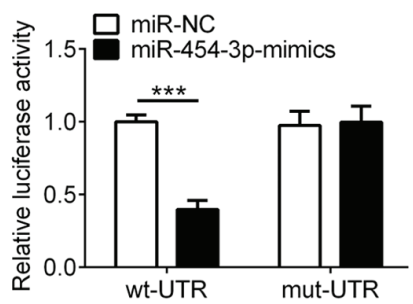

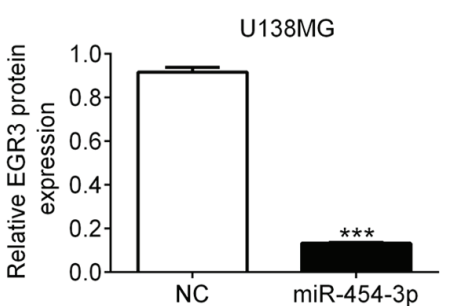

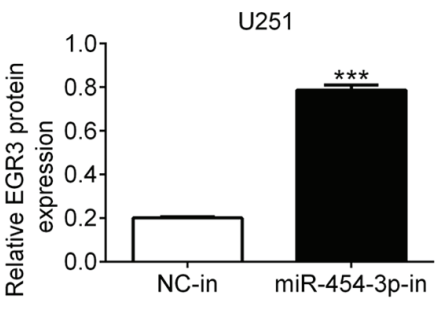

Figure 3. EGR3 is one target of miR-454-3p. (A) The predicted binding sites of miR-454-3p on the 3'-UTR of the EGR3 mRNA. (B) Relative luciferase activities of 293 T cells transfected with wild-type 3'UTR EGR3 (wt-UTR) or mutant of 3'-UTR (mut-UTR) and miR-454-3p mimics or NC. (C) Expression levels of EGR3 mRNA in U138MG cells transfected with miR-454-3p mimics and in U251 cells transfected with miR-454-3p inhibitor as determined using reverse transcription-quantitative PCR. (D) Expression of EGR3 protein in U138MG cells transfected with miR-454-3p mimics and in U251 cells transfected with miR-454-3p inhibitor as determined using western blotting. Representative images were shown and bands were quantified by densitometry and normalized against GAPDH. $n=3$. ${ }^{* * *} \mathrm{P}<0.001$. EGR3, early growth response 3; miR, microRNA; NC, negative control; 3'UTR, 3' untranslated region; wt, wild-type; mut, mutant.

miR-454-3p mimics increased cell viability $(n=5)$ and colony formation $(n=3)$, but was reversed by co-transfection with EGR3 plasmids in U138MG and U251 cells (Fig. 5C and D). These results indicated that miR-454-3p promote glioma cell proliferation in by negatively regulating EGR3 expression.

\section{Discussion}

Glioma is a common malignancy in the central nervous system with the overall prognosis of $\sim 1$ year for most patients from the time of diagnosis (20). Combined therapies currently available for glioma have limited benefits due to the high rates of invasion (21). It is therefore imperative to explore the pathological mechanism and new therapeutic strategies for the diagnosis and treatment of this disease.

A previous study have demonstrated that plasma levels of miR-454-3p were upregulated in patients with glioma compared with healthy controls, where the prognosis of glioma with high miR-454-3p expression was significantly worse compared with that of glioma with low miR-454-3p expression (22). Indeed, there is accumulating evidence that aberrant expression of miRNA is involved in the pathogenesis of many diseases including cancers (23). A number of studies have previously found that miR-454-3p was downregulated in gastric cancer and osteosarcomas $(23,24)$, whereas it was upregulated in prostate cancer, hepatocellular carcinoma and non-small cell lung cancer (25-27). These results suggest that expression profiles of miR-454-3p are distinct in different types of cancers. In the present study, it was found that miR-454-3p expression was upregulated in glioma tissues compared with adjacent normal tissues, which was concordant with previous results found in the plasma (22). In addition, association between increased miR-454-3p expression with poor prognosis were also previously found in hepatocellular carcinoma (26) and triple-negative breast cancer (28). Taken together, these results suggest that miR-454-3p may serve as a potential prognostic biomarker for glioma, though the role of miR-4543-3p in glioma remains unknown.

Since cell proliferation is one of the most important hallmarks of cancer pathophysiology (11), the present study evaluated the effect of miR-454-3p on glioma cell growth and found that miR-454-3p overexpression could significantly promote cell growth. EGR3 was subsequently identified as a direct target of miR-454-3p, where the overexpression of miR-454-3p inhibited EGR3 mRNA and protein expression. Overexpression of EGR3 was also found to reverse miR-454-3p mimic-induced cell proliferation in vitro. Previous studies reported that EGR3 was ubiquitously expressed in the cerebral cortex, substrate ganglion and neuromuscular spindle (13). Increased EGR3 expression induced expression of IL-6 and IL- 8 has been previously demonstrated in prostate cancer (29), suggesting that EGR3 also serves an important role in tumorigenesis. Of note, EGR3 were 
A
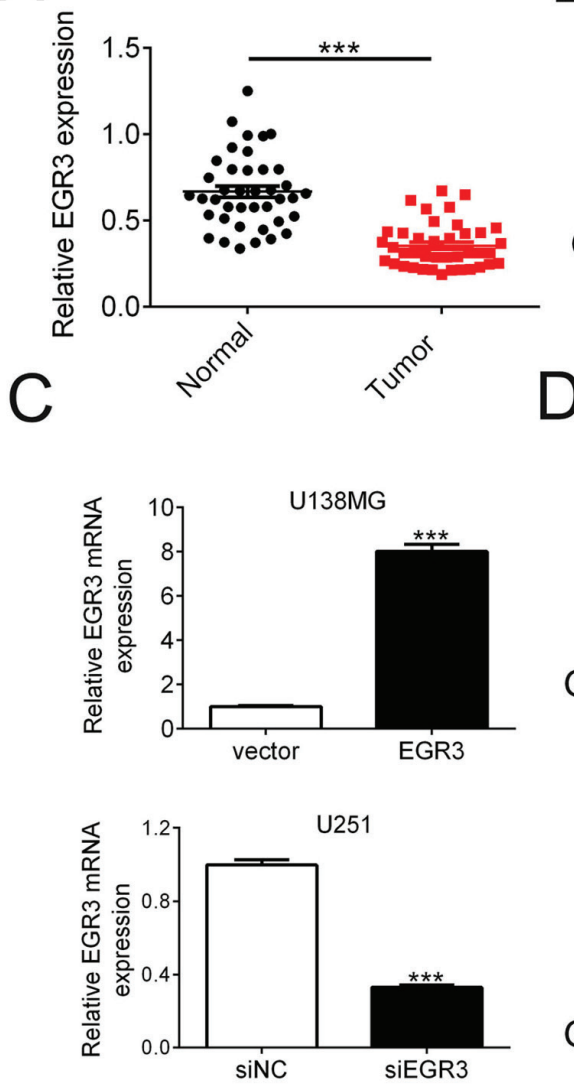

$E$
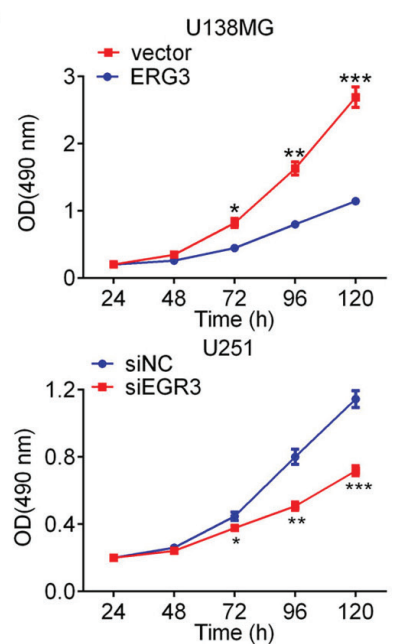

$\mathrm{F}$
B

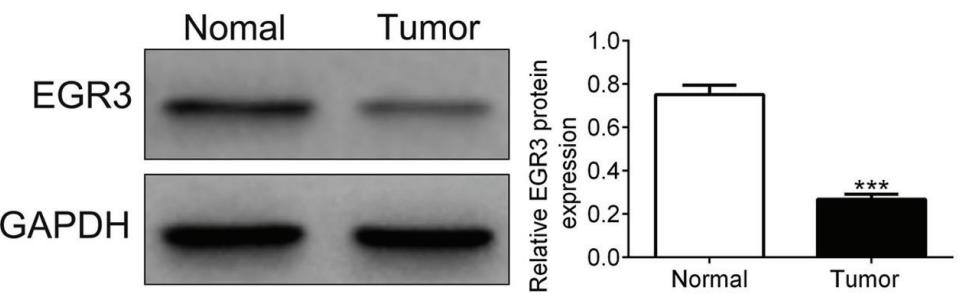

U138MG
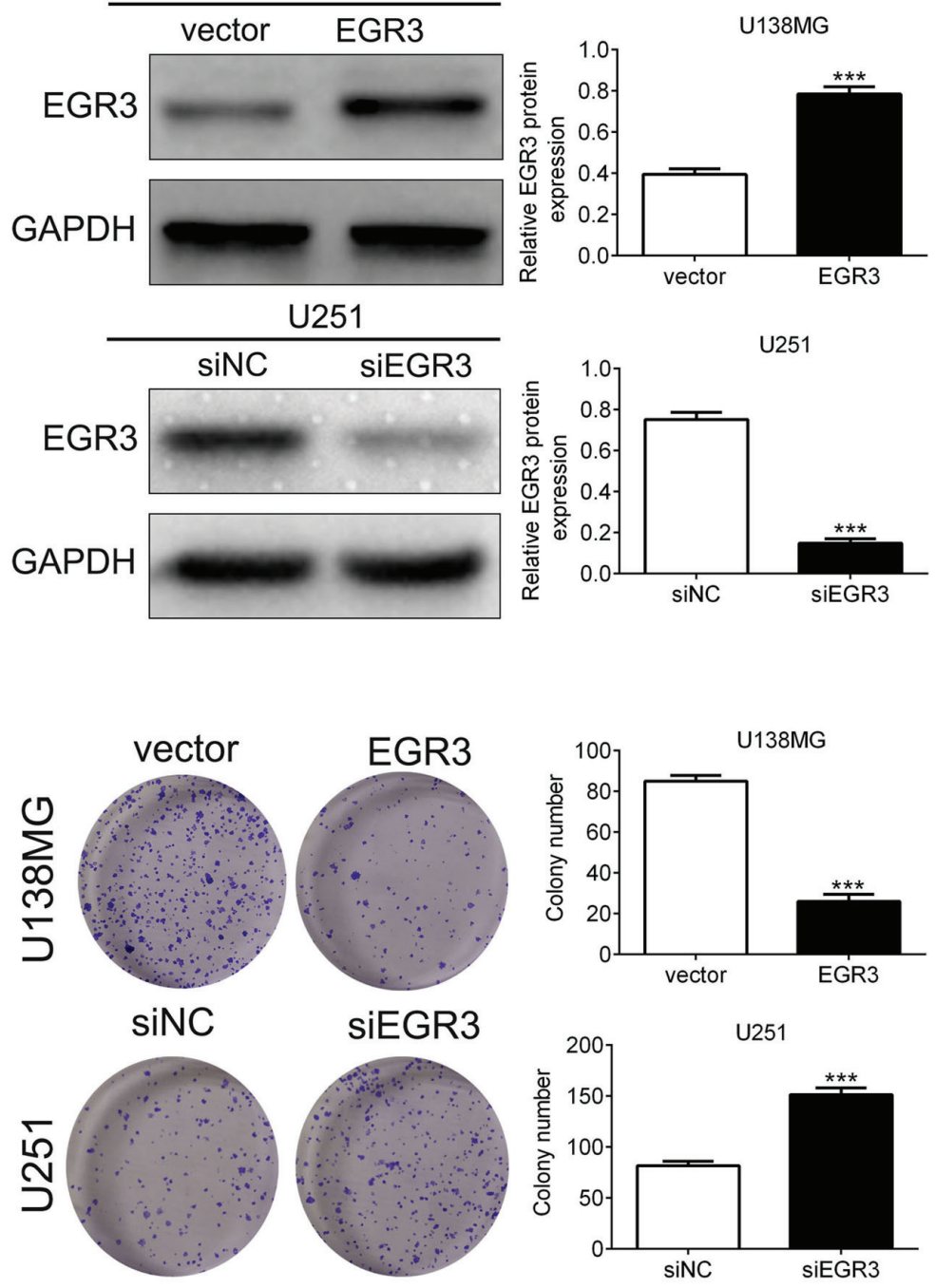

Figure 4. Cell proliferation in glioma following the upregulation or knockdown of EGR3 expression. Expression levels of EGR3 in glioma and adjacent normal tissues were determined using (A) RT-qPCR and (B) western blot analysis. (C) Expression levels of EGR3 mRNA in U138MG cells transfected with EGR3 plasmids and U251 cells transfected with EGR3 siRNA. (D) Expression levels of EGR3 protein in U138MG cells transfected with EGR3 plasmids and U251 cells transfected with EGR3 siRNA. (E) Cell viability in U138MG cells transfected with EGR3 plasmids and U251 cells transfected with EGR3 siRNA measured over five days using MTT assay. (F) Measurement of colony formation in U138MG cells transfected with EGR3 plasmids and U251 cells transfected with EGR3 siRNA. Representative images and quantification of crystal violet-stained cell colonies were shown. ${ }^{*} \mathrm{P}<0.01,{ }^{* *} \mathrm{P}<0.05$ and ${ }^{* * * *} \mathrm{P}<0.001$. EGR3, early growth response 3; miR, microRNA; NC, negative control; si, small interfering RNA.

also shown to be downregulated in glioma tissues in comparison with adjacent normal tissues, and EGR3 knockdown in vitro promoted glioma cell proliferation.

It was previously found that overexpression of miR-454-3p in hepatic stellate cells by transfection with miR-454-3p mimics could deactivate the hepatic stellate cells by targeting
SMAD4, which is involved in transforming growth factor- $\beta$ signaling (30). Overexpression of miR-454-3p could also promote prostate cancer cell growth by targeting $\mathrm{N}$-myc downstream-regulated gene 2 (25), whereas the upregulation miR-454-3p expression promoted the progression of NSCLCs by directly targeting PTEN (27). These results suggest that 
A

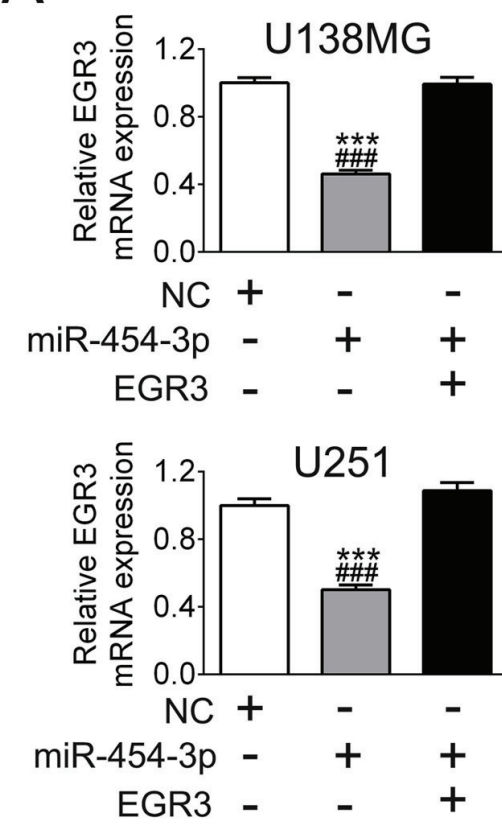

B

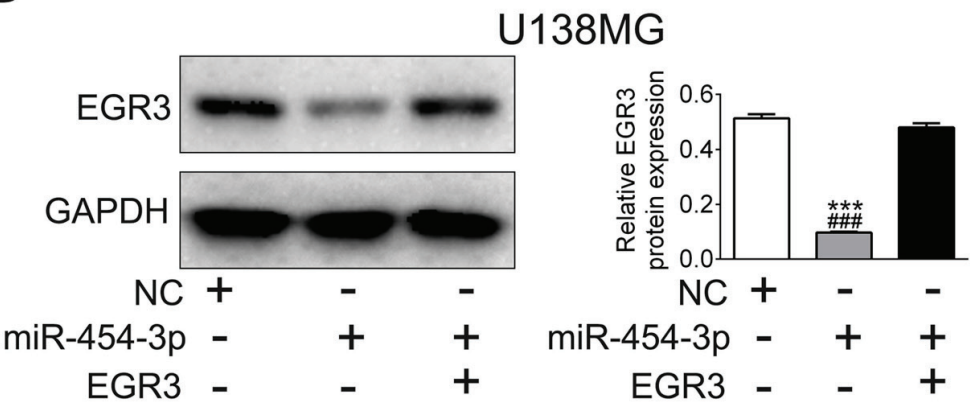

U251

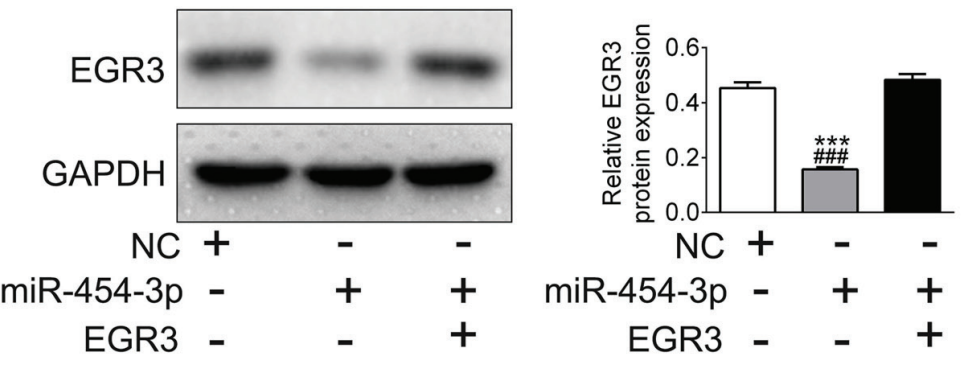

C

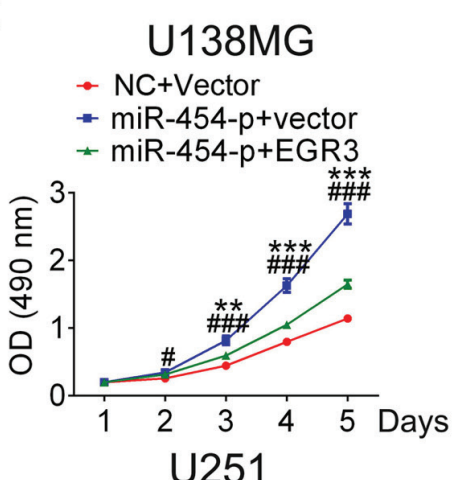

D

- NC+Vecto

- miR-454-p+vector

- miR-454-p+EGR3
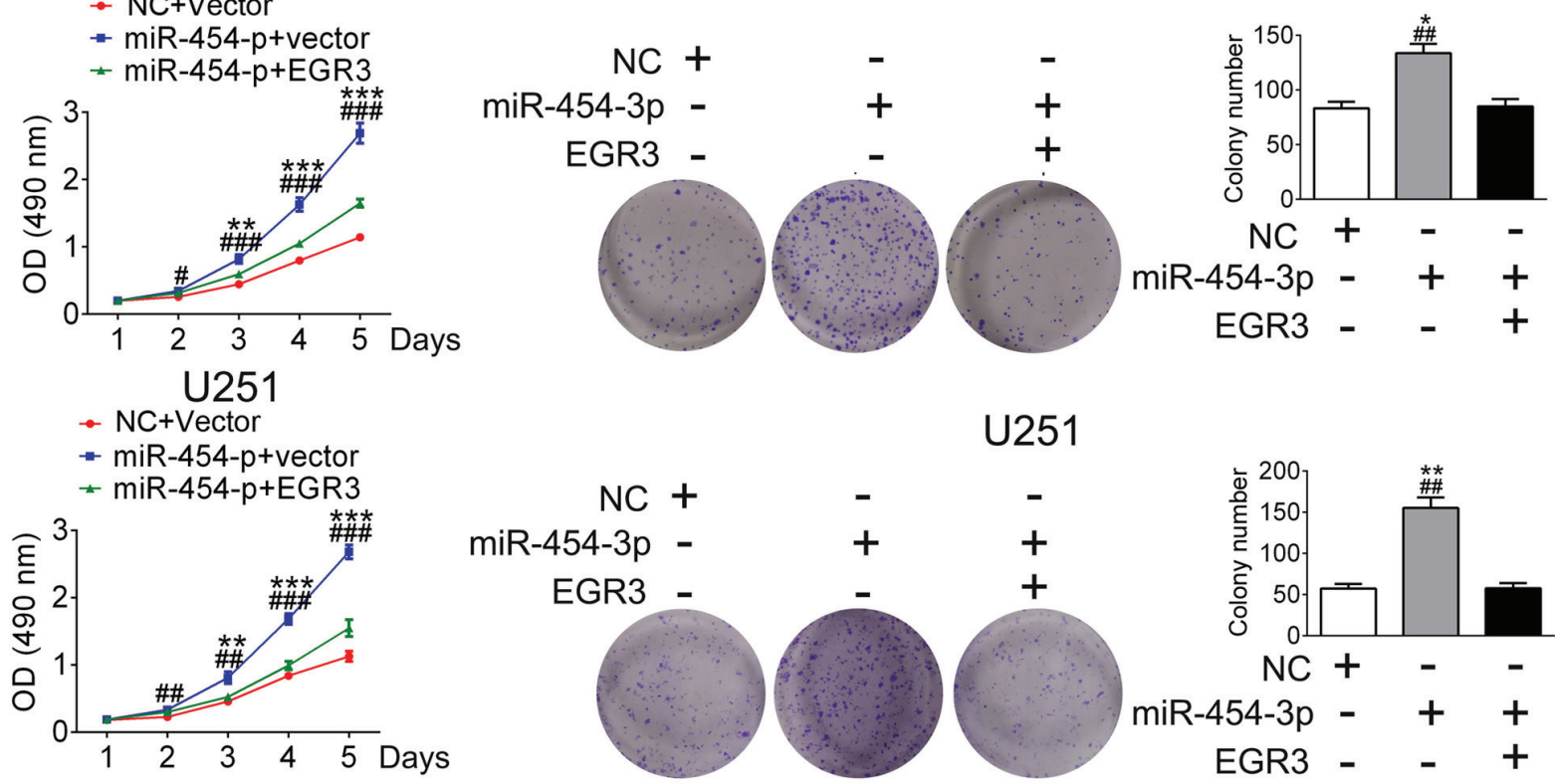

Figure 5. Glioma cell proliferation induced by miR-454-3p mimics was reversed by EGR3 overexpression. Expression levels of (A) EGR3 mRNA and (B) EGR3 proteins in U138MG and U251 cells transfected with miR-454-3p mimics only or co-transfected with miR-454-3p mimics and EGR3 plasmids. (C) Cell viability of U138MG and U251 cells transfected with miR-454-3p mimics only or co-transfected with miR-454-3p mimics and EGR3 plasmids as measured using MTT assays. (D) Measurement of colony formation in U138MG and U251 cells transfected with miR-454-3p mimics only or co-transfected with miR-454-3p mimics and EGR3 plasmids. Representative images and quantification of crystal violet-stained cell colonies were shown. ${ }^{*} \mathrm{P}<0.01,{ }^{* * *} \mathrm{P}<0.05$ and ${ }^{* * * *} \mathrm{P}<0.001$ vs. miR-454-3p + EGR3; ${ }^{\# P}<0.05$, ${ }^{\# \#} \mathrm{P}<0.01$ and ${ }^{\# \# "} \mathrm{P}<0.001$ vs. NC + vector. EGR3, early growth response 3; miR, microRNA; NC, negative control; si, small interfering RNA; OD, optical density.

miR-454-3p is involved in the regulation of a number of signaling pathways by binding to multiple targets. Therefore, the role of miR-454-3p in glioma may also be through other signaling pathway, which require further study.

To conclude, the present study was the first to demonstrate that miR-454-3p is in involved in the cell proliferation of glioma, by negatively regulating EGR3 expression. These findings provide valuable insights into the underlying biological features of this disease and may provide an avenue for the development of possible novel therapeutic targets.

\section{Acknowledgements}

Not applicable. 


\section{Funding}

No funding was received.

\section{Availability of data and materials}

All data generated or analyzed during this study are included in this published article.

\section{Authors' contributions}

GS and JW designed all the experiments and revised the paper. ZM, DC, LL and XL performed the experiments and wrote the paper.

\section{Ethics approval and consent to participate}

The present study was approved by the Research Ethics Committee of The First People's Hospital of Taizhou. All patients provided written informed consent prior to enrollment in the study.

\section{Patient consent for publication}

All patients provided written informed consent prior to enrollment in the study.

\section{Competing interests}

The authors declare that they have no competing interests.

\section{References}

1. Pan Y, Yuan F, Li Y, Wang G, Lin Z and Chen L: Bromodomain PHD-finger transcription factor promotes glioma progression and indicates poor prognosis. Oncol Rep 41: 246-256, 2019.

2. Yan J, Xu C, Li Y, Tang B, Xie S, Hong T and Zeng E: Long non-coding RNA LINC00526 represses glioma progression via forming a double negative feedback loop with AXL. J Cell Mol Med 23: 5518-5531, 2019

3. Guo Y, Hong W, Wang X, Zhang P, Körner H, Tu J and Wei W: MicroRNAs in microglia: How do micrornas affect activation, inflammation, polarization of microglia and mediate the interaction between microglia and glioma? Front Mol Neurosci 12: 125, 2019.

4. Petrescu GED, Sabo AA, Torsin LI, Calin GA and Dragomir MP MicroRNA based theranostics for brain cancer: Basic principles. J Exp Clin Cancer Res 38: 231, 2019.

5. Si W, Shen J, Zheng H and Fan W: The role and mechanisms of action of microRNAs in cancer drug resistance. Clin Epigenetics 11: 25, 2019.

6. Mohr AM and Mott JL: Overview of microRNA biology. Semin Liver Dis 35: 3-11, 2015.

7. Omar HA, El-Serafi AT, Hersi F, Arafa ESA, Zaher DM, Madkour M, Arab $\mathrm{HH}$ and Tolba MF: Immunomodulatory MicroRNAs in cancer: Targeting immune checkpoints and the tumor microenvironment. FEBS J: Jul 15, 2019 (Epub ahead of print) doi: 10.1111/febs. 15000 .

8. Ye X, Wei W, Zhang Z, He C, Yang R, Zhang J, Wu Z, Huang Q and Jiang Q: Identification of microRNAs associated with glioma diagnosis and prognosis. Oncotarget 8: 26394-26403, 2017.

9. Zhang Y, Zhang R, Sui R, Chen Y, Liang H, Shi J and Piao H MicroRNA-374a governs aggressive cell behaviors of glioma by targeting prokineticin 2 . Technol Cancer Res Treat 18 $1533033818821401,2019$.

10. Barciszewska AM: MicroRNAs as efficient biomarkers in high-grade gliomas. Folia Neuropathol 54: 369-374, 2016.

11. Liang HL, Hu AP, Li SL, Xie JP, Ma QZ and Liu JY: MiR-454 prompts cell proliferation of human colorectal cancer cells by repressing CYLD expression. Asian Pac J Cancer Prev 16: 2397-2402, 2015.
12. Piwecka M, Rolle K, Belter A, Barciszewska AM, Żywicki M, Michalak M, Nowak S, Naskręt-Barciszewska MZ and Barciszewski J: Comprehensive analysis of microRNA expression profile in malignant glioma tissues. Mol Oncol 9: 1324-1340, 2015.

13. Meyers KT, Marballi KK, Brunwasser SJ, Renda B, Charbel M, Marrone DF and Gallitano AL: The immediate early gene Egr3 is required for hippocampal induction of Bdnf by electroconvulsive stimulation. Front Behav Neurosci 12: 92, 2018.

14. Chien MH, Lee WJ, Yang YC, Li YL, Chen BR, Cheng TY, Yang PW, Wang MY, Jan YH, Lin YK, et al: KSRP suppresses cell invasion and metastasis through miR-23a-mediated EGR3 mRNA degradation in non-small cell lung cancer. Biochim Biophys Acta Gene Regul Mech 1860: 1013-1024, 2017.

15. Maple A, Lackie RE, Elizalde DI, Grella SL, Damphousse CC, Xa C, Gallitano AL and Marrone DF: Attenuated late-phase Arc transcription in the dentate gyrus of mice lacking Egr3. Neural Plast 2017: 6063048, 2017.

16. Oliveira Fernandes M and Tourtellotte WG: Egr3-dependent muscle spindle stretch receptor intrafusal muscle fiber differentiation and fusimotor innervation homeostasis. J Neurosci 35: 5566-5578, 2015.

17. Zhang S, Xia C, Xu C, Liu J, Zhu H, Yang Y, Xu F, Zhao J, Chang Y and Zhao Q: Early growth response 3 inhibits growth of hepatocellular carcinoma cells via upregulation of Fas ligand. Int J Oncol 50: 805-814, 2017.

18. Livak KJ and Schmittgen TD: Analysis of relative gene expression data using real-time quantitative PCR and the 2(-Delta Delta C(T)) method. Methods 25: 402-408, 2001.

19. Agarwal V, Bell GW, Nam JW and Bartel DP: Predicting effective microRNA target sites in mammalian mRNAs. Elife 4: $\mathrm{e} 05005,2015$.

20. Zhang Y, Chen J, Xue Q, Wang J, Zhao L, Han K, Zhang D and Hou L: Prognostic significance of MicroRNAs in glioma: A systematic review and meta-analysis. Biomed Res Int 2019: 4015969, 2019.

21. Beadle C, Assanah MC, Monzo P, Vallee R, Rosenfeld SS and Canoll P: The role of myosin II in glioma invasion of the brain. Mol Biol Cell 19: 3357-3368, 2008.

22. Shao N, Wang L, Xue L, Wang R and Lan Q: Plasma miR-454-3p as a potential prognostic indicator in human glioma. Neurol Sci 36: 309-313, 2015.

23. Kabekkodu SP, Shukla V, Varghese VK, Adiga D, Vethil Jishnu P, Chakrabarty $\mathrm{S}$ and Satyamoorthy K: Cluster miRNAs and cancer: Diagnostic, prognostic and therapeutic opportunities. Wiley Interdiscip Rev RNA: e1563, 2019.

24. Wang X, Liu B, Wen F and Song Y: MicroRNA-454 inhibits the malignant biological behaviours of gastric cancer cells by directly targeting mitogen-activated protein kinase 1 . Oncol Rep 39: 1494-1504, 2018.

25. Fu Q, Gao Y, Yang F, Mao T, Sun Z, Wang H, Song B and Li X: Suppression of microRNA-454 impedes the proliferation and invasion of prostate cancer cells by promoting $\mathrm{N}$-myc downstream-regulated gene 2 and inhibiting $W N T / \beta$-catenin signaling. Biomed Pharmacother 97: 120-127, 2018.

26. Zhou L, Qu YM, Zhao XM and Yue ZD: Involvement of miR-454 overexpression in the poor prognosis of hepatocellular carcinoma. Eur Rev Med Pharmacol Sci 20: 825-829, 2016.

27. Zhu DY, Li XN, Qi Y, Liu DL, Yang Y, Zhao J, Zhang CY, Wu K and Zhao S: MiR-454 promotes the progression of human non-small cell lung cancer and directly targets PTEN. Biomed Pharmacother 81: 79-85, 2016.

28. Cao ZG, Li JJ, Yao L, Huang YN, Liu YR, Hu X, Song CG and Shao ZM: High expression of microRNA-454 is associated with poor prognosis in triple-negative breast cancer. Oncotarget 7: 64900-64909, 2016.

29. Zhang P, Yang X, Wang L, Zhang D, Luo Q and Wang B: Overexpressing miR-335 inhibits DU145 cell proliferation by targeting early growth response 3 in prostate cancer. Int J Oncol 54: 1981-1994, 2019.

30. Zhu D, He X, Duan Y, Chen J, Wang J, Sun X, Qian H, Feng J, Sun W, Xu F and Zhang L: Expression of microRNA-454 in TGF- $\beta 1$-stimulated hepatic stellate cells and in mouse livers infected with Schistosoma japonicum. Parasit Vectors 7: 148, 2014.

This work is licensed under a Creative Common Attribution-NonCommercial-NoDerivatives 4.0 International (CC BY-NC-ND 4.0) License. 